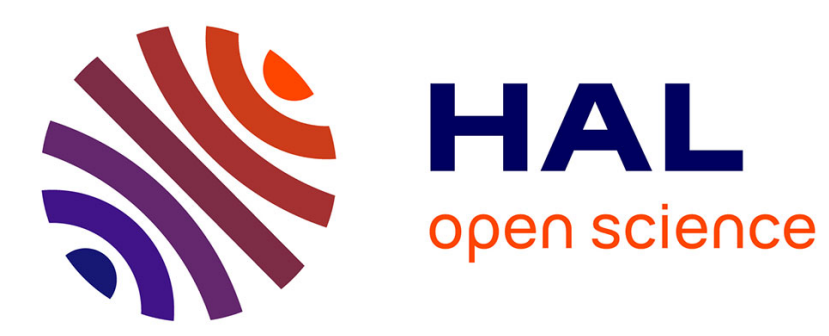

\title{
Modular and Reconfigurable Rectenna Unitcells with Beam-Forming Properties
}

E. Vandelle, Tan-Phu Vuong, Gustavo Ardila, Simon Hemour, Ke Wu

\section{To cite this version:}

E. Vandelle, Tan-Phu Vuong, Gustavo Ardila, Simon Hemour, Ke Wu. Modular and Reconfigurable Rectenna Unitcells with Beam-Forming Properties. 2019 49th European Microwave Conference (EuMC), Oct 2019, Paris, France. pp.109-112, 10.23919/EuMC.2019.8910889 . hal-02527666

\section{HAL Id: hal-02527666 \\ https://hal.science/hal-02527666}

Submitted on 1 Apr 2020

HAL is a multi-disciplinary open access archive for the deposit and dissemination of scientific research documents, whether they are published or not. The documents may come from teaching and research institutions in France or abroad, or from public or private research centers.
L'archive ouverte pluridisciplinaire HAL, est destinée au dépôt et à la diffusion de documents scientifiques de niveau recherche, publiés ou non, émanant des établissements d'enseignement et de recherche français ou étrangers, des laboratoires publics ou privés. 


\title{
Modular and Reconfigurable Rectenna Unitcells with Beam-Forming Properties
}

\author{
E. Vandelle ${ }^{1}$, Tan-Phu Vuong ${ }^{1}$, Gustavo Ardila ${ }^{1}$, Simon Hemour ${ }^{2}, \mathrm{Ke} \mathrm{Wu}^{3}$ \\ ${ }^{1}$ Université Grenoble Alpes, CNRS, Grenoble INP, IMEP-LAHC, Grenoble, France \\ ${ }^{2}$ Université de Bordeaux, IMS Bordeaux, Bordeaux Aquitaine INP, Bordeaux, France \\ ${ }^{3}$ Polytechnique Montréal, Poly-Grames, Montreal, Quebec, Canada
}

\begin{abstract}
This paper presents a modular and reconfigurable rectenna unitcell (RU) made up of an antenna, a rectifier and radiofrequency (RF) power combiners intended to form a scalable and adaptative beam-forming network, for autonomous passive tracking or RFID monitoring applications. The reconfigurable rectenna unitcell allows a dual mode operation thanks to both RF and DC possible combinations of multiple rectenna unitcells. On the one hand, the passive beam-forming operation, coming from the RF combination of multiple rectenna unitcells provides oriented communication or localization capabilities. On the other hand, the series DC connection of RF isolated rectenna unitcells delivers DC power to the system with high output voltage under low RF power densities. Prototypes of 4-edges rectenna unitcells are designed in the $2.4 \mathrm{GHz}$ band. The operation of one individual $\mathrm{RU}$ is demonstrated, and it is shown that multiple RUs are suitable for RF energy harvesting, multiple-tag communication and tag localization.
\end{abstract}

Index Terms-Rectenna, modular, scalable, localization, RF energy harvesting, RFID, beam-forming.

\section{INTRODUCTION}

The explosion of the number of electronics devices intended to be connected to the Internet imposes the development of low-cost and power-constrained communication networks aiming at battery-less operations. For instance, the technique of backscattering has permitted the development of passive radio frequency (RF) identification (RFID) tags, which are the basis of low-power wireless sensor networks in short range communications [1]. In this context, energy harvesting from ambient RF sources or Wireless Power Transfer (WPT) has gained a lot of attention for the energy supply of power-constrained communication networks [1], [2]. Multi-function systems integrating the energy-harvesting function can benefit from a prolonged lifetime and less maintenance.

In an effort of developing a multi-function system for autonomous RFID operations, this paper proposes a rectenna unitcell (RU) with collaborative passive beam-forming and energy harvesting capabilities. The passive beam-forming operation coming from the RF combination of multiple rectenna unitcells, along with the envelope detection (mode 1) provides oriented communication or localization capabilities. Beam-forming is an efficient technique to exchange information in a specific direction with narrow power beams [3]. It is generally used in communication since the reduction of the radiated beam widths along with the increase in gain allow for a high SINR and low interferences The use of passive beam-forming networks (BFNs) allows interactions between a reader and several tags at the same time due to the multiple ports [4]. Besides, if only one tag is tracked, this technique permits its localization with low power consumption In a second mode, the series DC connection of RF isolated rectenna unitcells is used to harvest ambient RF energy. Despite the low ambient power levels, the association of multiple RUs is a scalable system that permits to reach the DC power and DC voltage necessary for the targeted applications [5]

The principle and structure of the modular and reconfigurable RU allowing for the dual mode operation is presented in section II. Section III demonstrates the energy harvesting operation of one rectifying unitcell functioning in the $2.4 \mathrm{GHz}$ band and the output rectified voltage and power of series DC connected rectenna unitcells are given. Section IV demonstrates the feasibility of simultaneous communications between a 2-rectenna-unitcells-prototype and 2 tags and also shows the potential of the prototyped device for localization.

\section{RECTENNA UNITCELL'S PRINCIPLE}

The purpose of the proposed modular design is to ensure beam-forming capabilities for communication and localization functions along with the energy harvesting process to reach battery-less operations. As depicted in Figure 1, one layer of the RU is dedicated to a rectifying cell itself made up of halfhybrid couplers and one serial-diode rectifier while the other layer is the antenna part.

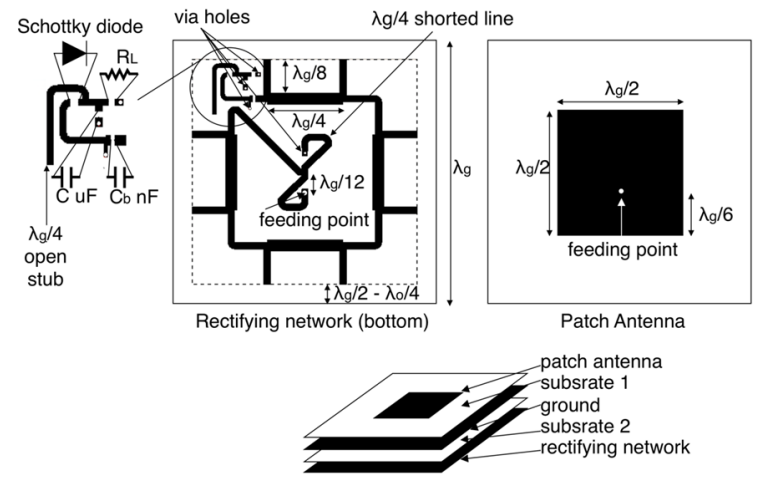

Fig. 1. Rectenna unitcell. 
The single serial diode rectifier, connected to the $50 \Omega$ half-hybrid couplers, is composed of a $10-\mathrm{nF}$ DC block capacitor followed by a short-circuited-stub matching network and the SMS-7630 Schottky diode. The load is composed of an output capacitor and a resistor, whose values vary with the operation mode of the cell (energy harvesting or envelope detection). The key element of the rectifying unitcell that allows the switching mode between RF and DC combinations is the half-hybrid couplers along each of the edges. The halfhybrid couplers allow the formation of a passive beamforming network (BFN) when several unitcells are associated together (RF connected), otherwise; when RF isolated, they act as $50 \Omega$ transmission lines. Since one half-hybrid coupler is assigned to each edge of the unitcell, the number of edges determines the maximum number of connected cells, which is equivalent to the number of beams created. Therefore, the maximum number of beams is equal to $2 n$ where $n$ is the number of connected half-hybrid couplers per cell. Figure 2 presents the different configurations of BFN that can be obtained with 4-edges unitcells, with different assemblies of 2 , 4 and 16 unitcells resulting in 2, 4 and 16 beams respectively. Note that up to 4 connected unitcells, additional connections the couplers at the extremities must be made.
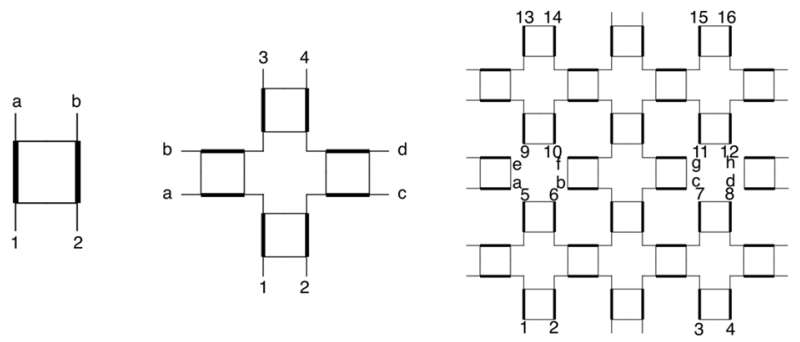

Fig. 2. Block diagrams of different possible configurations of the adaptative beam-forming network with a 4-edges rectenna unitcell. The numbers and letters represent the input and output ports.

When the RF combination is performed, each beam corresponding to the input of one unitcell is steered in the direction $\left(\theta_{s}, \phi_{s}\right)$ defined by the phasehifts and spacing between the rectenna unitcells (antennas). The steering angles are given by

$$
\begin{gathered}
\theta_{s}=\sin ^{-1}\left(\sqrt{\left(\frac{\beta_{x}}{d_{x}}\right)^{2}+\left(\frac{\beta_{y}}{d_{y}}\right)^{2}}\right) \\
\phi_{s}=\tan ^{-1}\left(\frac{\beta_{x} d_{x}}{\beta_{y} d_{y}}\right)
\end{gathered}
$$

In the case of 24 -edges rectenna unitcells RF connected along the $\mathrm{x}$-axis, the steering angles are $\beta_{x}=+/-90^{\circ}$ and $\beta_{y}=$ $0{ }^{\circ}$ resulting, according to (1) and (2), in 2 beams steered in the theoritical directions $\theta_{s}=+/-45^{\circ}$ and $\phi_{s}=0^{\circ}$ when the antenna are separated by $\lambda_{o} / 2$. The association of 4 RUs results in phaseshits $\beta_{x}=+1-90^{\circ}$ and $\beta_{y}=0^{\circ}$ leading to 4 theoritical steering angles $\theta_{s}=+/-45^{\circ}$ and $\phi_{s}=+/-45^{\circ}$ with a $\lambda_{o} / 2$ spacing between the antennas. In the case where up to 16 unitcells are put together, the steering angles are equally distributed along a circle in the azimuthal plane theoretically defined at $\theta_{s}=+/-45^{\circ}$.

The rectenna unit cells are intented to operate in two modes. In the first mode, the unitcells are RF connected and dedicated to short-range communications with multiple tags at different locations. As shown in Figure 3, in this mode, each rectifier is used as an enveloppe detector to demodulate the signal coming from a specific orientation determined by (1) and (2), that can be attributed to a tag. In the second mode, the unitcells are RF isolated and all the rectifiers are connected in series to harvest RF power and convert it into DC power. The optimal load of all the rectifiers in series is found by the DCDC converter with a maximum power point tracking, and approximately corresponds, at low power levels, to the junction resistance of the diode multiplied by the number of rectifiers. The DC-DC converter boosts and regulates the rectennas' output voltage so that energy can be supplied to a $\mu$-controller, responsible for switching between the two modes and for the information decoding.

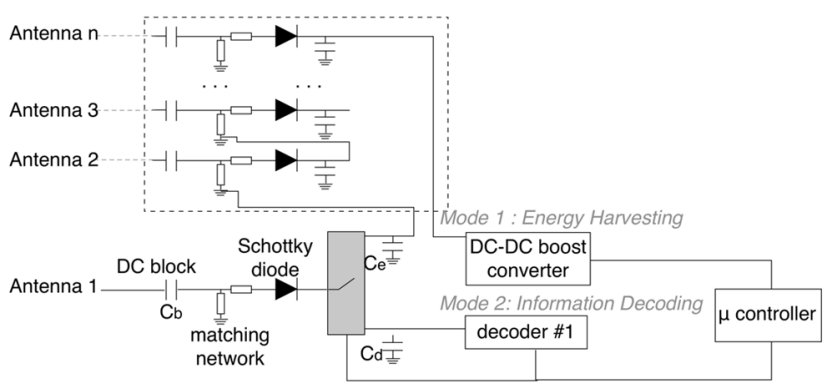

Fig. 3. Block diagram of the dual mode operation of the rectenna unitcells.

As a proof-of-concept, this work tests the rectenna unitcells for energy harvesting and envelope detecting, separately. Figure 4 shows the rectifying network of 1, 2 and 4 4-edges rectenna unitcells operating at $2.4 \mathrm{GHz}$. The rectifying cells are fabricated on the RO6002 laminate of thickness $0.762 \mathrm{~mm}$ and relative permittivity 2.94 .

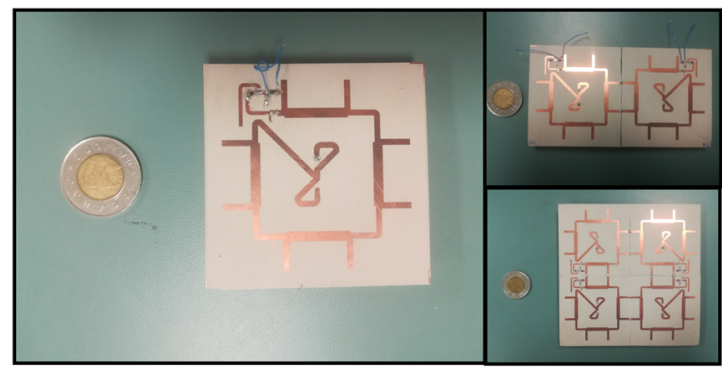

Fig. 4. Fabricated rectifying network of 1,2 and 4 rectenna unitcells, RF connected, operating in the $2.4 \mathrm{GHz}$ band.

\section{RF ENERGY HARVESTING FUNCTION}

In the RF energy harvesting process, the rectenna unitcells are RF isolated and all the rectifiers are connected in series as shown in Figure 3. All the diodes are connected to an output 


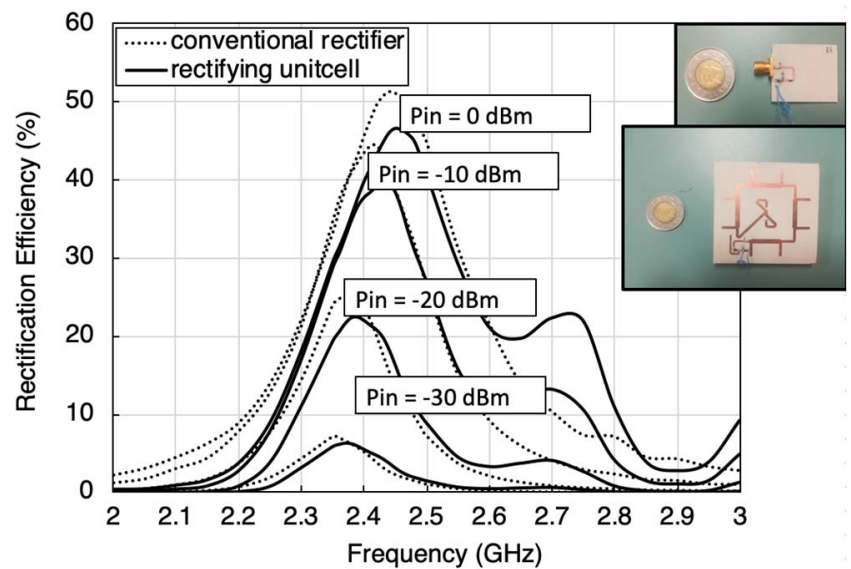

Fig. 5. Measured rectification efficiency (\%) of the rectifying unitcell compared to that of a conventional rectifier with a load of $5.1 \mathrm{k} \Omega$.

capacitor of $2.2 \mathrm{nF}$ that smooth the rectified power. The resistive load is chosen by the DC-DC converter to be proportional to the junction resistance of the diode $(5 \mathrm{k} \Omega)$ depending on the number or cells for optimal RF to DC conversion efficiency $\eta_{r}[6]$ defined as

$$
\eta_{r}=\frac{V_{D C}{ }^{2}}{R_{L} \cdot P_{R F}} \cdot 100 \%
$$

$V_{D C}$ is the DC voltage a the output of the rectifier across the load $R_{L}$ and $\mathrm{P}_{R F}$ is the input RF power. The experimental rectification efficiency of one single rectifying unitcell for a load $R_{L}=5.1 \mathrm{k} \Omega$ is plotted in Figure 5 as a function of the frequency for different input powers and compared to the efficiency of a conventional single serial diode rectifier fabricated on the same substrate. The rectification efficiency of the rectenna unitcell is satisfactory at low and high-power levels, which makes the rectenna unitcell suitable for harvesting RF power from ambient signals or intentional sources. A small frequency shift and slight insertion losses are observed compared to the rectification efficiency of a conventional serial diode rectifier, which can be caused by the open-circuit- and short-circuit-ended transmission lines present in the individual rectifying cell. The insertion losses are very limited at low power levels and becomes more significant with the increase of the input power. The output DC voltage is measured at the output of 1, 2 and 4 rectenna unitcells in an anechoic chamber. The patch antennas are fabricated on the RO6002 laminate of thickness $1.575 \mathrm{~mm}$. The RF power is delivered to the emitting antenna by a signal generator connected to a $30-\mathrm{dB}$ amplifier to produce ambient power densities, lower than $1 \mu \mathrm{W} \cdot \mathrm{cm}^{-2}$, at the system level. The power density at the receiving antenna level is first determined for various emitted RF powers at $2.4 \mathrm{GHz}$ by measuring, with a power meter, the power received by an antenna with a well-known aperture. Then, the output DC voltage is measured at the output of 1,2 and 4 rectenna unitcells, placed at the receiving position, across a resistive load proportional to the number of cells (5.1, 10 and $20 \mathrm{k} \Omega$ for 1,2 and 4 rectenna unitcells respectively) for optimal

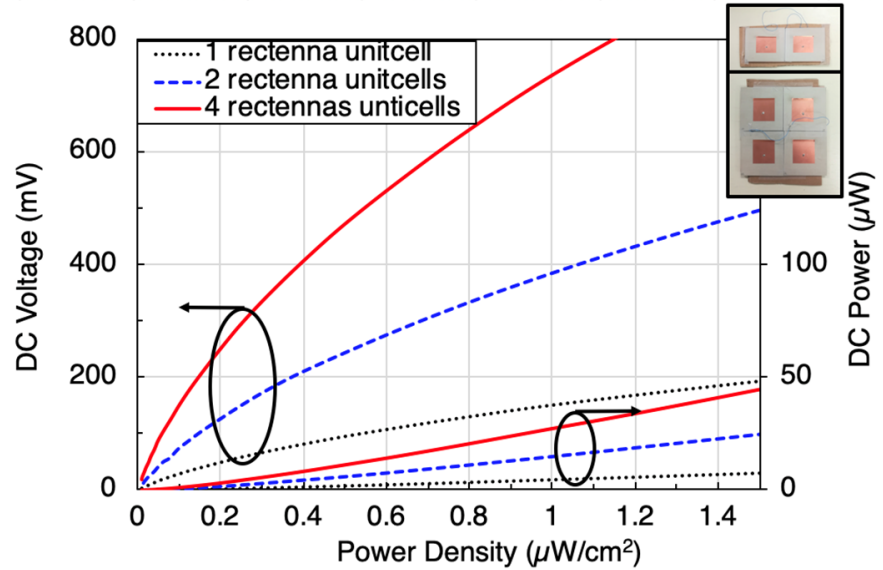

Fig. 6. Measured DC output voltage $(\mathrm{mV})$ and $\mathrm{DC}$ power $(\mu \mathrm{W})$ as a function of the available power density $\left(\mu \mathrm{w} . \mathrm{cm}^{-2}\right)$ of rectennas made up of 1,2 and 4 rectenna unitcells in series connection with a resistive load of 5.1, 10 and 20 $\mathrm{k} \Omega$ respectively.

rectification efficiency at low power levels [6]. Figure 6 shows that $\mathrm{DC}$ voltages of 150,395 and $728 \mathrm{mV}$, corresponding to DC powers of $4.3,17.8$ and $26.5 \mu \mathrm{W}$, are obtained at the output of 1, 2 and 4 unitcells respectively at a power density of $1 \mu \mathrm{W} . \mathrm{cm}^{-2}$. The DC voltage at the output of several RUs is approximately the product between the DC voltage delivered by a single RU and the number of RUs. Thus, the series connexion benefits the activation of the DC-DC converter, necessary to boost and regulate the rectified voltage. Besides, the scalability of the system allows us to choose the number of unitcells according to the amount of desired DC power/voltage. The entire document must be mainly in Times New Roman or Times font. Other fonts (e.g. Symbol font), if needed for special purposes, may be used sparingly. Type 3 fonts must not be used.

\section{SIMULTANEOUS COMMUNICATION AND LOCALIZATION FUNCTIONS}

The activation of the communication and localization functions requires the RF connection of all the RUs in a planar configuration and that the rectifiers act as enveloppe detectors. Thus, the diode of each cells is connected to a small capacitor and a high resistive load of $0.01 \mathrm{pF}$ and $220 \mathrm{k} \Omega$, respectively, for the demodulation of signals transmitted in the $2.4 \mathrm{GHz}$ band. In order to validate the communication capability of rectenna unitcells that associates beam-forming and demodulation process, a patch antenna, imitating a tag, transmits an AM modulated signal at $2.4 \mathrm{GHz}$ to the RUs. As a proof-of-concept, the experimental manipulation does not involve encoded data. As shown in Figure 7, a signal generator that produces a $0-\mathrm{dBm}$ signal with the OOK modulation is connected to the emitting patch antenna. The emitting antenna and the 2 RF connected RUs (RU2 and RU3) are placed in a small area, equivalent to a $33-\mathrm{cm}$ high animal cage to demonstrate the utility of the proposed system in the monitoring of laboratory animals for instance. The demodulated signal at the different outputs of the rectenna unitcells are observed on an oscilloscope for different 


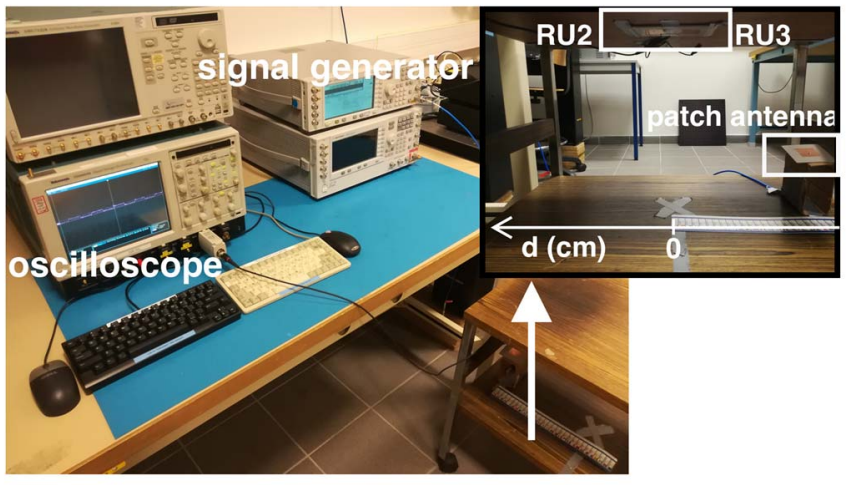

Fig. 7. Laboratory experimental setup.

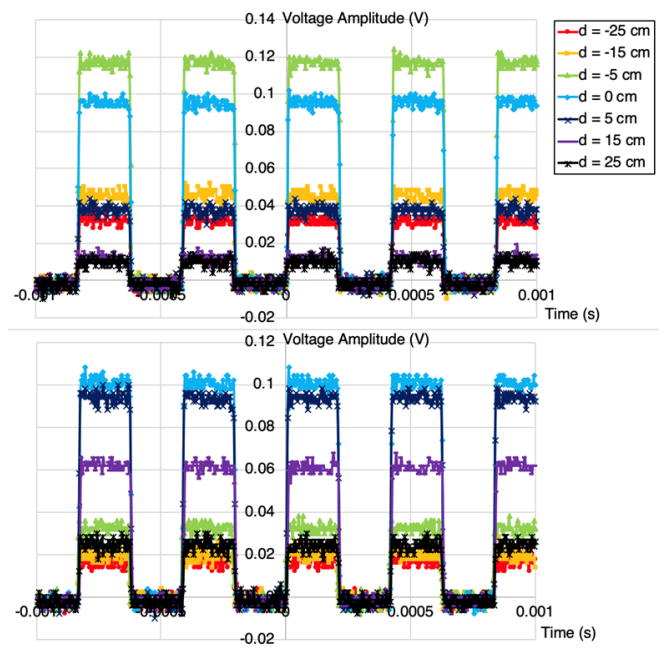

Fig. 8. Measured received and demodulated signal at the output of the two rectenna unitcells RF connected (RU2 on top and RU3 at the bottom) for different distances $d$ of the tag from the center of the cage (in front of the reader) to its edges.

locations of the emitting patch. Figure 8 presents the recorded signals for different distances $d$ of the patch antenna from the center of the box, which is positioned right beneath the RUs. It can be observed that while the unitcell RU2 is delivering higher output voltages when the emitting patch is on the right side of the box, RU3 produces higher voltages for left-sided locations of the patch. Figure 9 illustrates that result, which suggests that the unitcells could communicate with two tags in different positions in the areas 1 and 2 (right and left side of the box) without interference. Note that the number of controlled areas can be increased by increasing the number of RUs. Finally, the bottom graph of Figure 9 shows that, by computing the difference of the highest voltage at the output of RU2 and RU3, the distance $\mathrm{d}$ along one axis could be retrieved. This results also suggests that with a higher number of RUs in a planar configuration, a 2D localization can be performed within a range of $\lambda$, whose accuracy increases with the number of cells.

\section{CONCLUSION}

In this work, a modular rectenna unitcell was designed at $2.4 \mathrm{GHz}$ to propose a solution for both communication/localization and energy harvesting functions for autonomous

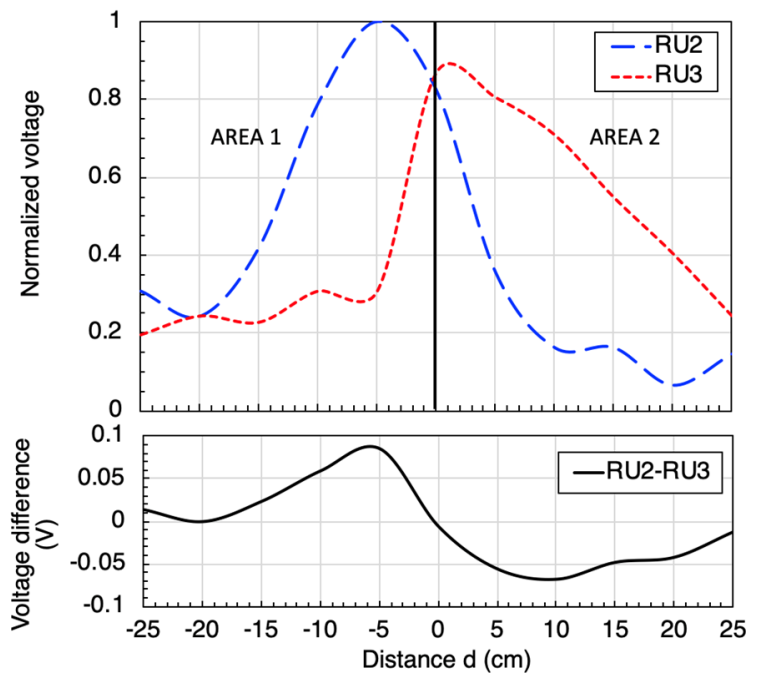

Fig. 9. Normalized voltage at the output of RU2 and RU3 (top graph) and voltage difference between the output of RU2 and RU3 (bottom graph) as a function of the distance $d$ of the tag from the center of the cage.

tracking or monitoring. The energy harvesting function was validated with experimental DC powers of $4.3,17.8$ and 26.5 $\mu \mathrm{W}$ at the output of a system made up of 1,2 and 4 unitcells and at a power density of $1 \mu \mathrm{W} \cdot \mathrm{cm}^{-2}$. Furthermore, a 2 rectenna unitcell was tested in a small area equivalent to an animal cage to demodulate an incoming signal produced by another antenna, which imitates a passive tag. It was shown that simultaneous communications with two tags in two different locations is possible. Furthermore, the system shows a great potential to localize an isolated tag.

\section{ACKNOWLEDGMENT}

The authors would like to thank IDEX for the EV travel grant and the region Rhône-Alpes for supporting the SCUSI project.

\section{REFERENCES}

[1] N. Van Huynh, D. T. Hoang, X. Lu, D. Niyato, P. Wang and D. I. Kim, "Ambient Backscatter Communications: A Contemporary Survey," in IEEE Communications Surveys \& Tutorials, vol. 20, no. 4, pp. 28892922, Fourthquarter 2018.

[2] A. Costanzo et al., "Energy Autonomous UWB Localization," in IEEE Journal of Radio Frequency Identification, vol. 1, no. 3, pp. 228-244, Sept. 2017.

[3] Y. Alsaba, S. K. A. Rahim and C. Y. Leow, "Beamforming in Wireless Energy Harvesting Communications Systems: A Survey," in IEEE Communications Surveys \& Tutorials, vol. 20, no. 2, pp. 1329-1360, Secondquarter 2018.

[4] P. Salonen and L. Sydanheimo, "A $2.45 \mathrm{GHz}$ digital beam-forming antenna for RFID reader," Vehicular Technology Conference. IEEE 55th Vehicular Technology Conference. VTC Spring 2002 (Cat. No.02CH37367), Birmingham, AL, USA, 2002, pp. 1766-1770 vol.4.

[5] Z. Popović et al., "Scalable RF Energy Harvesting," in IEEE Transactions on Microwave Theory and Techniques, vol. 62, no. 4, pp. 1046-1056, April 2014.

[6] S. Hemour, Y. Zhao, C.H.P. Lorenz, D. Houssameddine, Y. Gui, C-M $\mathrm{Hu}, \mathrm{K}$. Wu, "Towards Low-Power High-Efficiency RF and Microwave Energy Harvesting," IEEE Trans. Microw. Theory Tech., vol. 62, no. 4, pp. 965-976, Apr. 2014. 